\title{
Changing Tendency Analysis of Sleep Quality on the Basis of Temperature and Respiratory Frequency Using a Neural Network Model
}

\author{
Minami Tsuchiya D, Seung-II Cho (D, Atsushi Tanaka D, Muneki Yasuda D, \\ Tomochika Harada iD, and Michio Yokoyama
}

Graduate School of Science and Engineering, Yamagata University, Yonezawa, Japan

\section{]jfis}

\begin{abstract}
This study investigates the invisible factors that influence the subjective satisfaction of sleep, which is a crucial factor in sleep-supporting systems. The effects of these factors were examined using a neural network (NN) model. A sensing bed system obtains the environmental and vital signs data with five-level evaluations. The relationships among over 300,000 collected data points were visualized and analyzed using the NN model. The results indicated that the temperature difference between the inside and outside of the bed affect the subjective satisfaction of sleep both during warm and cold periods. Furthermore, the NN models indicated that the respiratory frequency influences sleep satisfaction. In this study, both these factors and their effects on the subjective satisfaction of sleep were successfully investigated by utilizing the NN model.
\end{abstract}

Keywords: Neural network model, Temperature, Respiratory frequency, Sleep quality, Environmental, Vital data sensing

\section{Introduction}

The quality of sleep is considered to be deteriorating in recent times. Many people feel dissatisfied with their quality of sleep [1]. To improve the quality of sleep, the environmental factors and/or vital signals during sleep, which contribute to sleep satisfaction, should be identified [2, 3]. However, these parameters and their effects on the subjective satisfaction of sleep for different time periods have not yet been sufficiently clarified. Evaluation of the

Received: Jan. 17, 2020 Revised : May 29, 2020 Accepted: Jun. 1, 2020

Correspondence to: Michio Yokoyama (yoko@yz.yamagata-u.ac.jp)

(OThe Korean Institute of Intelligent Systems

(c) This is an Open Access article distributed under the terms of the Creative Commons Attribution Non-Commercial License (http://creativecommons.org/licenses/ by-nc/3.0// which permits unrestricted noncommercial use, distribution, and reproduction in any medium, provided the original work is properly cited. subjective satisfaction of sleep is an aspect that is yet to be considered. For instance, even if a person states that their subjective satisfaction of sleep is high, their response might be motivated by their condition slightly prior to waking up. It is difficult to detect how the subjective satisfaction of sleep changes within the sleep duration. Hence, changes in the subjective satisfaction of sleep must be investigated.

This study aims to reveal the factors affecting the subjective satisfaction of sleep using a neural network (NN) model [4, 5]. It attempts to analyze the time-change tendency of these factors. The rest of this paper is organized as follows. Sleep-related studies are introduced in Section 2. The experimental conditions, including the sensors used and an overview of the analysis, are detailed in Section 3. Subsequently, the analysis method using the NN model and some formulas are described in Section 4. Two aspects of the analyzed results are discussed in Section 5. Finally, we present our conclusions in Section 6. 


\section{Related Works}

Several researchers have analyzed the various factors related to sleep and proposed sleep monitoring systems with the aim of attaining high sleep quality. There are some reports on the correlation between tossing and turning during sleep and sleep stage transitions, such as rapid eye movement (REM) sleep, non-REM sleep, and awakening [6]. It is well known that tossing and turning play a role in the changes in humidity and temperature inside a bed. These are also reported to be conditions that affect the quality of sleep [7]. For instance, it has been commercialized that smart mattresses and mattress pads adjust the bed temperature automatically [8]. In addition, the frequency of respiration is strongly correlated with sleep satisfaction such that patients suffering from sleep apnea suffer from sleep problems such as frequent awakening [9]. An Internet-ofThings (IoT)-based unobtrusive sleep monitoring pillow was proposed to monitor breathing patterns and quantify the overall sleep quality [10]. To obtain the subjective satisfaction of sleep, objective and subjective evaluations were conducted on a sleeping environment [11]. It was revealed that the sleep satisfaction and overall thermal sensation were higher in rooms with a thermoelectric air duct cooling system than in naturally ventilated test rooms.

\section{Experiment and Analysis}

A healthy male in his 30 s participated in this preliminary sleep experiment, as illustrated in Figure 1. The study was conducted for 112 days spanning across four seasons in Japan. The subject slept in his bedroom and performed his daily routine according to his own rhythm, without any limitations on the starting time of his sleep or environment parameters, such as temperature and humidity. Therefore, the length of sleep was not constant.

The sensors used in this experiment were selected by referring to the parameters related to sleep evaluation that were reported in previous works [7--10], for example, thermometers, hygrometers, and barometer sensors to measure the temperature changes and humidity inside and outside the bed [7, 8]; microwave and piezo sensors to measure sleep apnea, tossing and turning [9]; and acceleration sensors to monitor the motion on the pillow [10]. Accelerometers, thermometers, and hygrometers were the sensors installed inside the bed. Meanwhile, thermometers, hygrometers, a barometer, and a microwave sensor were the sensors installed over the bed. A microcomputer system was developed to send the data from these sensors to the server. Acceleration, temperature $\left({ }^{\circ} \mathrm{C}\right)$, humidity $(\%)$, atmospheric pressure $(\mathrm{hPa})$, and body motion $(\%)$ were recorded at intervals of 10 seconds during the duration of sleep. The method of obtaining the heart rate and respiratory frequency is as follows: frequency analysis was performed on the heartbeat wave, respiration, and movement with MATLAB R2014b. The sampling frequency was $64 \mathrm{~Hz}$, window function was a Hamming window, window interval was 60 seconds, and overlap was 10 seconds. The heart rate and respiratory frequency were defined as maximum power spectrum density between 0.5-4.0 $\mathrm{Hz}$ and $0.1-2.0 \mathrm{~Hz}$, respectively. Subsequently, the sleep apnea syndrome index (SASI) was calculated as follows:

$$
\text { SASI }(\%)=\frac{\text { Time duration under } 0.3 \mathrm{~Hz} \text { of respiration }}{10 \text { minutes }} \times 100 \text {. }
$$

The data were collected over a period of 112 days, in increments of 10 seconds during the sleep cycle. In addition, a questionnaire was completed after the subject woke up, re-

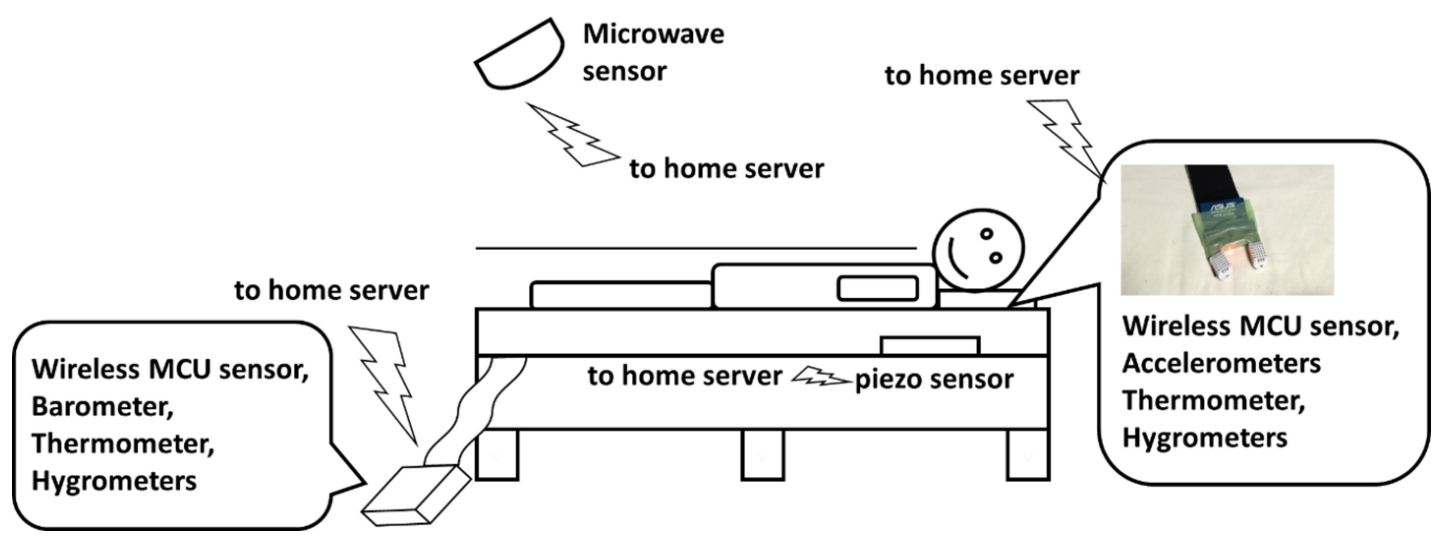

Figure 1. Experimental environment of this work. 
Table 1. Descriptions of 14 sensor parameters

\begin{tabular}{|c|c|c|c|c|}
\hline No. & Parameter & Unit & Sensor & Position \\
\hline 1 & Max_X & None & Accelerometers & Under the pillow \\
\hline 2 & $\operatorname{Max}_{-} \mathrm{Y}$ & None & Accelerometers & Under the pillow \\
\hline 3 & $\operatorname{Max} Z$ & None & Accelerometers & Under the pillow \\
\hline 4 & Atmosphere & $\mathrm{hPa}$ & A barometer & Outside the bed \\
\hline 5 & Room temperature (Tr) & ${ }^{\circ} \mathrm{C}$ & Thermometers & Outside the bed \\
\hline 6 & Temperature inside the bed $1(\mathrm{~Tb} 1)$ & ${ }^{\circ} \mathrm{C}$ & Thermometers & Under the pillow \\
\hline 7 & Temperature inside the bed 2 ( $\mathrm{Tb} 2)$ & ${ }^{\circ} \mathrm{C}$ & Thermometers & Under the pillow \\
\hline 8 & Room humidity (Hr) & $\%$ & Hygrometers & Outside the bed \\
\hline 9 & Humidity inside the bed 1 ( $\mathrm{Hb} 1)$ & $\%$ & Hygrometers & Under the pillow \\
\hline 10 & Humidity inside the bed 2 ( $\mathrm{Hb} 2$ ) & $\%$ & Hygrometers & Under the pillow \\
\hline 11 & Heart rate & $\mathrm{Hz}$ & Microwave sensor & Attached to ceiling, directed at the bed \\
\hline 12 & Respiratory frequency & $\mathrm{Hz}$ & Microwave sensor & Attached to ceiling, directed at the bed \\
\hline 13 & Motion & $\%$ & Piezo sensor & Under mattress \\
\hline 14 & Sleep apnea syndrome index & $\%$ & $\begin{array}{c}\text { None (calculated by } \\
\text { No.12) }\end{array}$ & - \\
\hline
\end{tabular}

garding the subjective satisfaction of sleep using a five-level evaluation. The data were added to the analysis factors.

The obtained data set was processed and analyzed by the NN model in Section 4. The proposed NN model was utilized to further examine the correlation between changing sensor parameters. The first parameter to be considered was the temperature, since it strongly influences sleep satisfaction according to the literature [7]. A total of 14 sensor parameters were selected from the obtained sensor data, and the daily average of these parameter values was considered to correspond to the daily subjective satisfaction score. Table 1 describes these 14 sensor parameters. We investigated changes in satisfaction by observing the fluctuation in individual sensor parameters against other steady parameters.

Prior to the application of the proposed NN method, we used a multiple regression analysis (MRA) to investigate how the changes in each sensor parameter with time affect the sleep satisfaction, as opposed to the use of daily subjective satisfaction of sleep. The environmental and vital signs data were obtained over the span of 112 days, recorded every 10 seconds. The data for 14 parameters were collected every 10 seconds to obtain the estimation formula for satisfaction. Changes in the estimated satisfaction, Tr, room humidity, and respiratory frequency were calculated as the difference between consecutive values. Figure 2 illustrates the comparison between the estimation using MRA and the actual subjective satisfaction as answered by the subject.
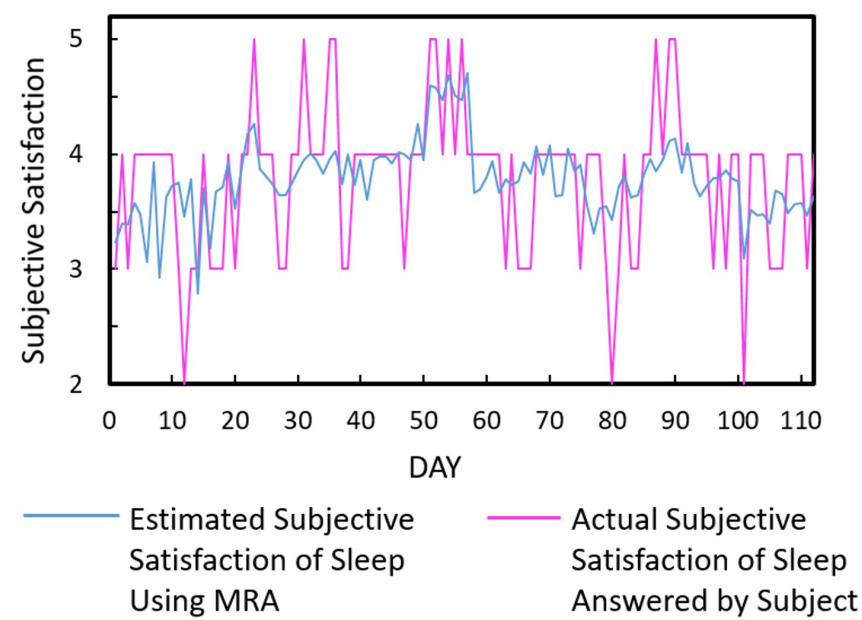

Figure 2. Comparison between estimation using MRA and actual subjective satisfaction answered by the subject.

The mean absolute error (MAE) was 0.451. This value is at least small enough considering that the sleep satisfaction of the subject does not change into level 2 when it is level 1.

\section{Neural Network for Our Analysis}

The NN model is used to examine the relationship between the sensor parameters and subjective sleep satisfaction level. To derive the sleep satisfaction level from the 14 sensor parameters, the NN model is utilized on the basis of a pattern recognition system. 


\subsection{Pattern Recognition System based on NN}

Consider a pattern recognition system constructed by an NN with the soft-max classifier, in which we want to classify a given input vector $\boldsymbol{x}=\left(x_{1}, x_{2}, \cdots, x_{n}\right)^{\mathrm{T}} \in \boldsymbol{R}^{n}$ into $K$ different classes, $c_{1}, c_{2}, \cdots, c_{K}$. We use the 1 -of- $K$ vector representation to identify each class, in which each class corresponds to the $K$-dimensional vector $\boldsymbol{t}=\left(t_{1}, t_{2}, \cdots, t_{n}\right)^{\mathrm{T}}$, the elements of which are $t_{k} \in\{0,1\}$ and $\sum_{k=1}^{K} t_{k}=1$, i.e., a vector in which only one element equals one and the remaining elements are zero. Hence, when $t_{k}=1, \boldsymbol{t}$ indicates class $c_{k}$. For simplicity of notation, we denote the 1-of- $K$ vector, the $k$-th element of which is one, by $1_{k}$. We denote $K$ outputs of the $\mathrm{NN}$ for an input $\boldsymbol{x}$ by $q_{1}(\boldsymbol{x}, \theta), q_{2}(\boldsymbol{x}, \theta), \ldots, q_{K}(\boldsymbol{x}, \theta)$, where $\theta$ is the set of learning parameters in the NN. By using $K$ outputs of the $\mathrm{NN}$, the soft-max classifier computes the class probabilities as

$$
\begin{aligned}
& P(\boldsymbol{t} \mid \boldsymbol{x}, \theta):=\frac{1}{Z(\boldsymbol{x}, \theta)} \exp \left(\sum_{k=1}^{K} q_{k}(\boldsymbol{x}, \theta) t_{k}\right), \\
& \boldsymbol{t} \in\left\{1_{k} \mid k=1,2, \ldots, K\right\}
\end{aligned}
$$

where

$$
Z(\boldsymbol{x}, \theta):=\sum_{k=1}^{K} \exp \left(q_{k}(\boldsymbol{x}, \theta)\right)
$$

is the normalization constant (see Figure 3(a)). The pattern recognition system computes all the class probabilities, $P\left(\mathbf{1}_{1} \mid\right.$ $\boldsymbol{x}, \theta), P\left(\mathbf{1}_{2} \mid \boldsymbol{x}, \theta\right), \ldots, P\left(\mathbf{1}_{K} \mid \boldsymbol{x}, \theta\right)$, according to Eq. (2) and classifies the input into the class with the maximum probability.

The learning parameters in $\theta$ are tuned during the training with the training set composed of $N$ training data: $\boldsymbol{D}=$ $\left\{\left(\mathbf{x}^{(\mu)}, \mathbf{t}^{(\mu)} \mid \mu=1,2, \ldots, N\right\}\right.$, where $\mathbf{x}^{(\mu)}$ is $\mu$ th input vector

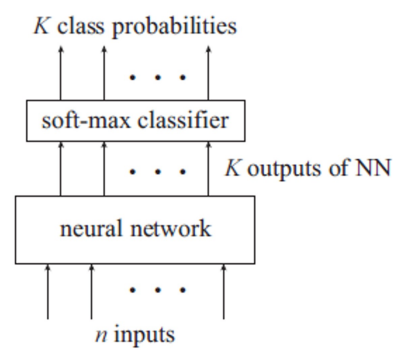

(a)

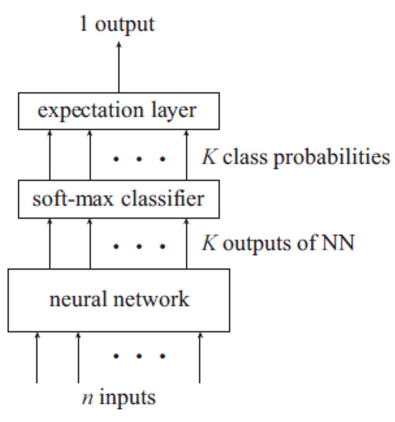

(b)
Figure 3. (a) Usual pattern recognition system and (b) proposed pattern recognition system with the expectation layer. The expectation layer outputs the expectation of the class values. and $\mathbf{t}^{(\mu)}$ is the corresponding supervised 1-of- $K$ vector, namely, the target class. In the training, the learning parameters are controlled to maximize $P\left(\mathbf{t}^{(\mu)} \mid \mathbf{x}^{(\mu)}, \theta\right)$ for all $\mu$. This can be achieved by minimizing the cross-entropy loss,

$$
E_{C E}(\theta):=-\frac{1}{N} \sum_{\mu=1}^{K} \sum_{k=1}^{K} t_{k}^{(\mu)} \ln P\left(\mathbf{1}_{k} \mid \boldsymbol{x}^{(\mu)}, \theta\right),
$$

with respect to $\theta$.

\subsection{Stacking Expectation Layer}

In the training mentioned in the previous section, the magnitude relationships among the class probabilities other than the target class are not explicitly considered. This is because of the lack of order relations (or a concept of closeness) among the classes. However, in some cases, we might want to introduce an order relation. In our sleep-satisfaction problem, the classes correspond to the satisfaction level of his or her sleep at five levels, $l=1,2,3,4,5$. The $k$-th class, $c_{K}$, corresponds to the satisfaction level of $l=k$ and, therefore, $K=5$ in this case. The satisfaction level of $l=4$ should be closer to that of $l=5$ than that of $l=3$. That is, when $P\left(\mathbf{1}_{5} \mid \boldsymbol{x}, \theta\right)$ is the largest, $P\left(\mathbf{1}_{4} \mid \boldsymbol{x}, \theta\right)$ should be larger than $P\left(\mathbf{1}_{3} \mid \boldsymbol{x}, \theta\right)$.

To introduce an order relation among the classes, we stack a new layer, referred to as the expectation layer, on the soft-max classifier (see Figure 3(b)). By using the class probabilities obtained from the soft-max classifier, the expectation layer computes a scaler output defined as

$$
y(\boldsymbol{x}, \theta):=\sum_{k=1}^{K} l_{k} P\left(\mathbf{1}_{K} \mid \boldsymbol{x}, \theta\right),
$$

where $l_{k} \in R$ is the class value assigned into $c_{K}$. The class values express the order relation among the classes and must be appropriately set by the designer. If we want to introduce the order relation such as $c_{1}<c_{2}<\cdots<c_{K}$, the class values should be set to $l_{1}<l_{2}<\cdots<l_{K}$. In our sleep-satisfaction problem, $l_{k}=k . y(\boldsymbol{x}, \theta)$ is regarded as the expectation of the class values. From the definition, $y(\boldsymbol{x}, \theta)$ lies in the interval $\min _{k} l_{k} \leq y(\boldsymbol{x}, \theta) \leq \max _{k} l_{k}$. Using an appropriate quantization of $y(\boldsymbol{x}, \theta)$, we can obtain the result of the classification. For example, we classify input $\mathbf{x}$ into the $k$ th class that minimizes $\left|l_{k}-y(\boldsymbol{x}, \theta)\right|$.

For the training set of the expectation layer, we propose the 
following error function:

$$
E(\theta):=\rho E_{M S E}(\theta)+(1-\rho) E_{C E}(\theta), \quad 0<\rho<1,
$$

where

$$
E_{M S E}(\theta):=\frac{1}{N} \sum_{\mu=1}^{N}\left(\sum_{k=1}^{K} l_{k} t_{k}^{(\mu)}-y\left(\boldsymbol{x}^{(\mu)}, \theta\right)\right)^{2}
$$

is the mean square error (MSE) between the class value of the target class and $y(\boldsymbol{x}, \theta)$, and $E_{C E}(\theta)$ is the usual crossentropy (CE) loss defined in Eq. (4). The pattern recognition system with the expectation layer is trained by minimizing the error function in Eq. (6) with respect to $\theta$. The hyperparameter $\rho$ in Eq. (6) controls the magnitude relationship between $E_{M S E}(\theta)$ and $E_{C E}(\theta)$. In our experiment, $\rho$ was set to 0.1. By minimizing of $E_{M S E}(\theta)$, it is expected that the class probability of the class, which is closer to the target class, is likely to be larger; that is, the desired order relation is obtained. The minimization of $E_{C E}(\theta)$ is also crucial in the elimination of unexpected results. Suppose that $K=5$, $l_{k}=k$, and $c_{3}$ is the target class for a certain input $\mathbf{x}$. Consider these two cases: (i) $P\left(\mathbf{1}_{1} \mid \boldsymbol{x}, \theta\right)=0, P\left(\mathbf{1}_{2} \mid \boldsymbol{x}, \theta\right)=0.25$, $P\left(\mathbf{1}_{3} \mid \boldsymbol{x}, \theta\right)=0.5, P\left(\mathbf{1}_{4} \mid \boldsymbol{x}, \theta\right)=0.25$, and $P\left(\mathbf{1}_{5} \mid \boldsymbol{x}, \theta\right)=0$ and (ii) $P\left(\mathbf{1}_{1} \mid \boldsymbol{x}, \theta\right)=0, P\left(\mathbf{1}_{2} \mid \boldsymbol{x}, \theta\right)=0.5, P\left(\mathbf{1}_{3} \mid \boldsymbol{x}, \theta\right)=0$, $P\left(\mathbf{1}_{4} \mid \boldsymbol{x}, \theta\right)=0.5$, and $P\left(\mathbf{1}_{5} \mid \boldsymbol{x}, \theta\right)=0$. Both these cases lead to the same $y(\boldsymbol{x}, \theta)$. The result of case (ii) is unexpected and should be eliminated.

\subsection{Perturbative Analysis of Output Response}

Assuming that the learning parameters in $\theta$ are tuned by minimizing Eq. (6) with an appropriate learning algorithm, we consider the response of $y(\boldsymbol{x}, \theta)$ for a small variation in $\mathbf{x}$. This helps us obtain a simple relation between the output and input. Consider

$$
\Delta_{i}(\boldsymbol{x}, \varepsilon, \theta):=y\left(\boldsymbol{x}+\boldsymbol{\varepsilon}_{i}, \theta\right)-y(\boldsymbol{x}, \theta),
$$

where $\varepsilon_{i} \in \boldsymbol{R}^{n}$ is the variation vector whose $i$-th element is $\varepsilon$ and the other elements are zero. Eq. (8) represents the variation of the output against a small variation of $x_{i}$. It is regarded as the response of the output for the small variation.

At approximately $\varepsilon=0$, we expand $y\left(\boldsymbol{x}+\boldsymbol{\varepsilon}_{i}, \theta\right)$ as

$$
y\left(\boldsymbol{x}+\boldsymbol{\varepsilon}_{i}, \theta\right)=y(\boldsymbol{x}, \theta)+\sum_{m=1}^{\infty} \frac{1}{m !} A_{i}^{(m)}(\boldsymbol{x}, \theta) \varepsilon^{m},
$$

where

$$
A_{i}^{(m)}(\boldsymbol{x}, \theta):=\lim _{\varepsilon \rightarrow 0} \frac{\partial^{m} y\left(\boldsymbol{x}+\boldsymbol{\varepsilon}_{i}, \theta\right)}{\partial \varepsilon^{m}}=\frac{\partial^{m} y(\boldsymbol{x}, \theta)}{\partial x_{i}^{m}} .
$$

For $m=1,2,3$, from Eqs. (5) and (10), we obtain

$$
\begin{aligned}
A_{i}^{(1)}(\boldsymbol{x}, \theta)= & \sum_{k=1}^{K}\left(l_{k}-y(\boldsymbol{x}, \theta)\right) P\left(\mathbf{1}_{k} \mid \boldsymbol{x}, \theta\right) \frac{\partial q_{k}(\boldsymbol{x}, \theta)}{\partial x_{i}}, \\
A_{i}^{(2)}(\boldsymbol{x}, \theta)= & \sum_{k=1}^{K}\left(l_{k}-y(\boldsymbol{x}, \theta)\right) P\left(\mathbf{1}_{k} \mid \boldsymbol{x}, \theta\right) \\
& \times\left\{\frac{\partial^{2} q_{k}(\boldsymbol{x}, \theta)}{\partial x_{i}^{2}}+\left(\frac{\partial q_{k}(\boldsymbol{x}, \theta)}{\partial x_{i}}\right)^{2}\right\} \\
& -2 A_{i}^{(1)}(\boldsymbol{x}, \theta) \sum_{k=1}^{K} P\left(\mathbf{1}_{k} \mid \boldsymbol{x}, \theta\right) \frac{\partial q_{k}(\boldsymbol{x}, \theta)}{\partial x_{i}} \\
A_{i}^{(3)}(\boldsymbol{x}, \theta)= & \sum_{k=1}^{K}\left(l_{k}-y(\boldsymbol{x}, \theta)\right) P\left(\mathbf{1}_{k} \mid \boldsymbol{x}, \theta\right) \\
& \times\left\{\frac{\partial^{3} q_{k}(\boldsymbol{x}, \theta)}{\partial x_{i}^{3}}+3 \frac{\partial q_{k}(\boldsymbol{x}, \theta)}{\partial x_{i}} \frac{\partial^{2} q_{k}(\boldsymbol{x}, \theta)}{\partial x_{i}^{2}}\right. \\
& \left.+\left(\frac{\partial q_{k}(\boldsymbol{x}, \theta)}{\partial x_{i}}\right)^{3}\right\} \\
& -3 A_{i}^{(1)}(\boldsymbol{x}, \theta) \sum_{k=1}^{K} P\left(\mathbf{1}_{k} \mid \boldsymbol{x}, \theta\right) \\
& \times\left\{\frac{\partial^{2} q_{k}(\boldsymbol{x}, \theta)}{\partial x_{i}^{2}}+\left(\frac{\partial q_{k}(\boldsymbol{x}, \theta)}{\partial x_{i}}\right)^{2}\right\} \\
& -3 A_{i}^{(2)}(\boldsymbol{x}, \theta) \sum_{k=1}^{K} P\left(\mathbf{1}_{k} \mid \boldsymbol{x}, \theta\right) \frac{\partial q_{k}(\boldsymbol{x}, \theta)}{\partial x_{i}}
\end{aligned}
$$

where $q_{k}(\boldsymbol{x}, \theta)$ is the output of the NN model appearing in Eq. (2) and its specifications depend on the structure of the NN model.

From Eqs. (8) and (9), we obtain

$$
\begin{aligned}
\Delta_{i}(\boldsymbol{x}, \varepsilon, \theta)= & A_{i}^{(1)}(\boldsymbol{x}, \theta) \varepsilon+\frac{1}{2} A_{i}^{(2)}(\boldsymbol{x}, \theta) \varepsilon^{2} \\
& +\frac{1}{6} A_{i}^{(3)}(\boldsymbol{x}, \theta) \varepsilon^{3}+O\left(\varepsilon^{4}\right) .
\end{aligned}
$$

For a given value of $x$ and $\theta$, by using the above equation and Eqs. (11)-(13), we can obtain the response of $y(\boldsymbol{x}, \theta)$ for any small $\varepsilon$ in a simple form in terms of the cubic function.

The SmoothGrad method proposed in [12] is a work related to our method. The SmoothGrad method observes the output response based on the first-order gradients of inputs, whereas our method observes it based on the first-, second-, and thirdorder gradients of the inputs. Our method allows the observation 
of more precise responses than the SmoothGrad method.

\subsection{Sleep Data Analysis with NN}

The obtained data were analyzed by using learning to identify the changes in sensor parameters, which contributed to changes in the estimated satisfaction. A three-layered NN model was used, as shown in Figure 4. The NN had an input, intermediate, and output layer consisting of 14, 7, and 5 nodes, respectively, namely, $n=14$ and $K=5$ in this case. The outputs of the NN model for a given input $x$ are represented as

$$
q_{k}(\boldsymbol{x}, \theta)=b_{k}^{(2)}+\sum_{j=1}^{7} w_{k, j}^{(2)} a\left(b_{j}^{(1)}+\sum_{i=1}^{n} w_{j, i}^{(1)} x_{i}\right),
$$

where $\left\{b_{k}^{(2)}\right\}$ and $\left\{b_{j}^{(1)}\right\}$ are the bias parameters and $\left\{w_{k, j}^{(2)}\right\}$ and $\left\{w_{j, i}^{(1)}\right\}$ are the weight parameters. $a(x)$ is the activation function of the first intermediate layer, in which the sigmoid function is adopted. In the preprocessing stage, all the input data, $\left\{x^{(\mu)}\right\}$, were normalized in the following manner:

$$
\hat{x}^{(\mu)} \leftarrow \frac{x_{i}^{(\mu)}-m_{i}}{\sqrt{v_{i}}},
$$

where

$$
\begin{aligned}
m_{i} & :=\frac{1}{N} \sum_{\mu=1}^{N} x_{i}^{(\mu)}, \\
v_{i} & :=\frac{1}{N} \sum_{\mu=1}^{N}\left(x_{i}^{(\mu)}-m_{i}\right)^{2} .
\end{aligned}
$$

By using the normalized input data, $\left\{\hat{x}^{(\mu)}\right\}$, the learning parameters, $\theta=\left\{b_{k}^{(2)}, b_{j}^{(1)}, w_{k, j}^{(2)}, w_{j, i}^{(1)}\right\}$, were trained by

\section{Three-layered Neural Network Model}

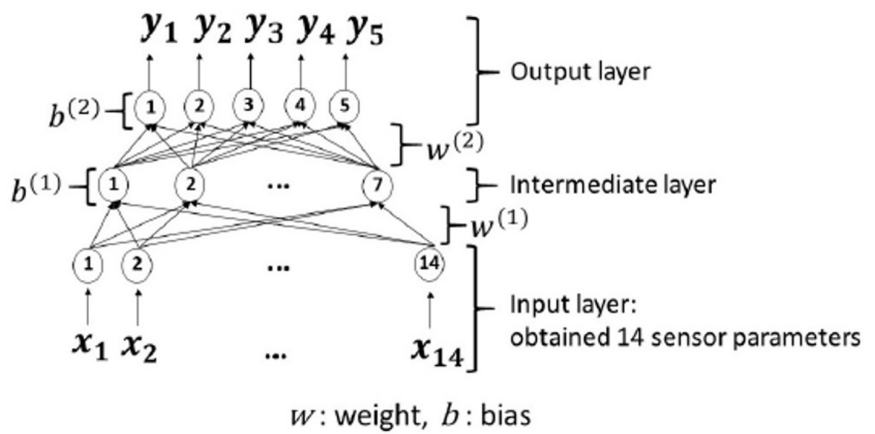

Figure 4. Structure of the three-layered NN model.

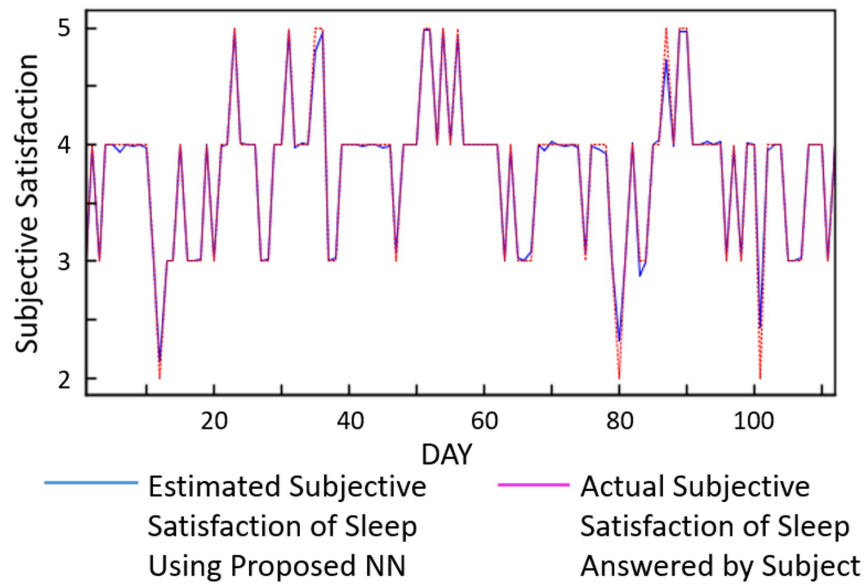

Figure 5. Comparison between the estimation using the proposed $\mathrm{NN}$ method and actual subjective satisfaction as answered by the subject.

minimizing the error function in Eq. (6). In the training, we used the momentum method used in [13]. Figure 5 shows the accuracy of the estimation of the NN model. The MAE was found to be 0.028 .

\section{Results of the NN Model}

\subsection{Results of Daily Parameters Using the NN Model}

In this study, the border between warm and cold period is defined as the average of $\operatorname{Tr}$ for 112 days, which is $22^{\circ} \mathrm{C}$. The relationship between each factor and sleep satisfaction was studied using the proposed NN model. We investigated the changes in satisfaction by observing the fluctuations of individual sensor parameters against other steady parameters. Figure 6 displays the results obtained by the NN model, which depicts changes in subjective satisfaction of sleep with fluctuations of $\mathrm{Tr}$ and $\mathrm{Tb}$ in the warm period and in the cold period. The blue and orange lines represent $\operatorname{Tr}$ and $\mathrm{Tb}$, respectively. Meanwhile, the vertical and horizontal axes represent the changes in subjective satisfaction for sleep and the fluctuation of $\mathrm{Tr}$ and $\mathrm{Tb}$, respectively. Zero on the $\mathrm{X}$-axis represents the average of $\mathrm{Tr}$ or $\mathrm{Tb}$ for each period. That is, zero for each axis represents the current state. Subsequently, we investigated the relative micro change to estimate the precise change using the NN model. The third decimal place is adopted.

As shown in Figure 6(a), the subjective satisfaction of sleep in the warm period increases when $\mathrm{Tb}$ decreases while $\mathrm{Tr}$ increases. As shown in Figure 6(b), with Tr below $22^{\circ} \mathrm{C}$ (hereinafter, referred to as the cold period), the subjective satisfaction 


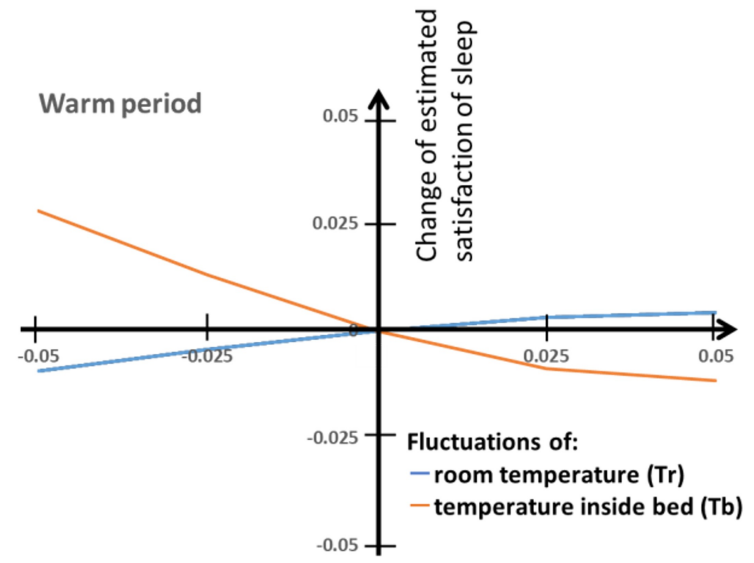

(a)

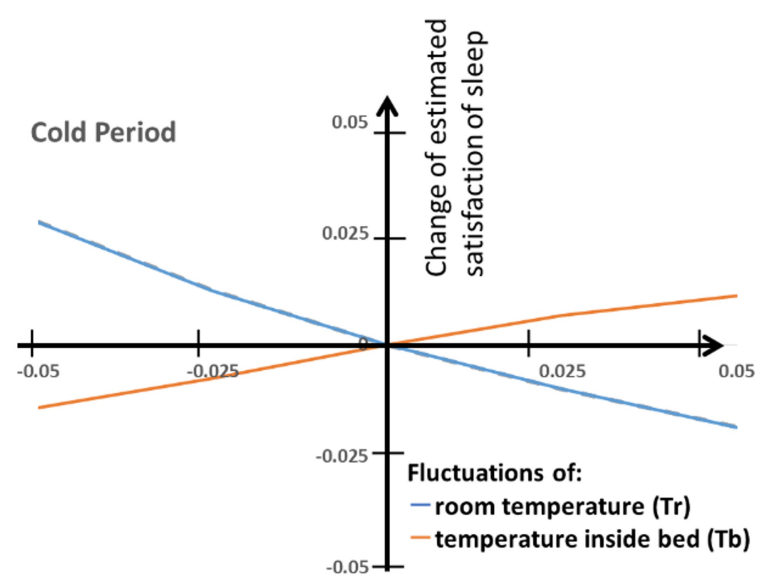

(b)

Figure 6. Change in the subjective satisfaction of sleep with fluctuation of $\mathrm{Tr}$ and $\mathrm{Tb}$ during (a) the warm period and (b) the cold period.

of sleep increases when Tb increases while Tr decreases.

This indicates that the subjective satisfaction of sleep increases when $\mathrm{Tb}$ is low and $\mathrm{Tr}$ is high in the warm period and decreases when $\mathrm{Tb}$ is low and $\mathrm{Tr}$ is high in the cold period. Hence, the difference between these two temperatures in the warm and cold periods is related to sleep satisfaction.

\subsection{Results Using the NN Model for Continuous Parame- ters}

Figure 7 displays the results obtained using the NN model. It exhibits the change in the subjective satisfaction of sleep with fluctuations in respiratory frequency. The solid line represents the estimated satisfaction. Meanwhile, the vertical and horizontal axes represent the changes in the estimated satisfaction of sleep and fluctuation of the respiration frequency, respectively.

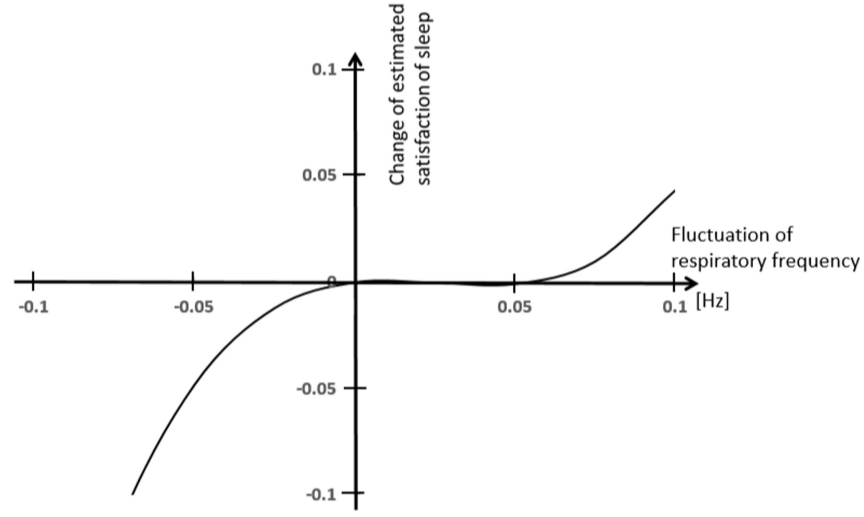

Figure 7. Change in subjective satisfaction of sleep with fluctuation of respiratory frequency.

As mentioned in Section 5.1, zero on each axis represents the current state. Subsequently, the relative micro-change was investigated using NN to estimate the change. The value is considered correct to the second decimal place. It is evident that the estimated satisfaction increases when the respiratory frequency increases. It is also revealed that the decrease in respiratory frequency significantly affects the decrease in the subjective satisfaction of sleep.

\section{Conclusion}

The proposed NN model was used to obtain the factors that affect the subjective satisfaction of sleep. An experiment was conducted using a sensing bed system for 112 days, spanning across four seasons in Japan. It was revealed that there was a higher tendency for subjective sleep satisfaction in warm periods, when the temperature inside the bed was lower than the room temperature. The tendency was observed to be vice versa in the cold period. Furthermore, application of the NN model with the continuously recorded data exhibited a correlation between an increase in the respiratory frequency and increase in sleep satisfaction. It was also shown that a decrease in respiratory frequency significantly decreases the estimated sleep satisfaction. Both these factors and their effects on the subjective satisfaction of sleep were successfully investigated in this study by utilizing the NN model.

Some future research aspects are as follows: a polysomnography study as an objective assessment for the comparison of the subjective satisfaction of sleep, which is the primary parameter in this study; an increase in the number of subjects; and further examination of the relationship between tossing-turning and the subjective satisfaction of sleep. 


\section{Conflict of Interest}

No potential conflict of interest relevant to this article was reported.

\section{Acknowledgments}

This work was partly supported by JSPS KAKENHI (Grant Numbers JP17H01729, JP17K01781, JP15K00330, and JP15H0 3699). It was also partially supported by the Center of Innovation Program from the Japan Science and Technology Agency (JST), and JST CREST (Grant Number JPMJCR1402), and MIC SCOPE (Strategic Information and Communications R\&D Promotion Programme) (Grant Number 172302009).

\section{References}

[1] Z. Wang, J. Ma, C. Miyoshi, Y. Li, M. Sato, Y. Ogawa, et al., "Quantitative phosphoproteomic analysis of the molecular substrates of sleep need," Nature, vol. 558, no. 7710, pp. 435-439, 2018. https://doi.org/10.1038/s41586018-0218-8

[2] M. Tsuchiya, A. Tanaka, M. Yasuda, T. Harada, and M. Yokoyama, "Evaluation of sleep quality based on environmental and vital sensor signals using big data analysis and deep learning," in Proceedings of the 2017 International Symposium on Nonlinear Theory and Its Applications (NOLTA), Cancun, Mexico, 2017, pp. 38-41.

[3] S. I. Cho, M. Yasuda, M. Tsuchiya, T. Harada, A. Tanaka, and M. Yokoyama, "Estimation of correlation between multiple parameters and sleep satisfaction using deep neural network for pleasant sleeping support system," in Proceedings of 31st International Conference on Computer Applications in Industry and Engineering (CAINE), New Orleans, LA, 2018, pp. 26-30.

[4] Y. Bengio, "Learning deep architectures for AI," Foundations and Trends in Machine Learning, vol. 2, no. 1, pp. 1-127, 2009. https://doi.org/10.1561/2200000006

[5] I. Goodfellow, Y. Bengio, and A. Courville, Deep Learning. Cambridge, MA: The MIT Press, 2016.

[6] S. Shirakawa, "The role and dynamic state in body movement at sleep," Brain Wave and Electromyogram, vol. 17, pp. 221-234, 1989.
[7] M. Miyazawa, "On the correlation between the seasonal changes of bed climate and sleep," Research Journal of Living Science, vol. 21, no. 1, pp. 99-106, 1974.

[8] K. Matsui, "Pleasant sleep provision system during warm nights as a function of smart home," in Proceedings of 2017 International Symposium on Networks, Computers and Communications (ISNCC), Marrakech, Morocco, 2017, pp. 1-6. https://doi.org/10.1109/ISNCC.2017. 8071991

[9] A. Bidarian-Moniri, M. Nilsson, J. Attia, and H. Ejnell1, "Mattress and pillow for prone positioning for treatment of obstructive sleep apnoea," Acta Oto-Laryngologica, vol. 135, no. 3, pp. 271-276, 2015. https://doi.org/10.3109/ 00016489.2014 .968674

[10] A. Veiga, L. Garcia, L. Parra, J. Lloret, and V. Augele, "An IoT-based smart pillow for sleep quality monitoring in AAL environments," in Proceedings of 2018 Third International Conference on Fog and Mobile Edge Computing (FMEC), Barcelona, Spain, 2018, pp. 175-180. https://doi.org/10.1109/FMEC.2018.8364061

[11] K. Irshad, A. I. Khan, S. Algarni, K. Habib, B. B. Saha, "Objective and subjective evaluation of a sleeping environment test chamber with a thermoelectric air cooling system," Building and Environment, vol. 141, 2018, pp 155-165. https://doi.org/10.1016/j.buildenv.2018.05.061

[12] D. Smilkov, N. Thorat, B. Kim, F. Viegas, and M. M. Wattenberg, "SmoothGrad: removing noise by adding noise," 2017, Available https://arxiv.org/pdf/1706.03825 pdf

[13] H. Peng, L. Mou, G. Li, Y. Liu, L. Zhang, and Z. Jin, "Building program vector representations for deep learning," in Knowledge Science, Engineering and Management. Cham: Springer, 2015, pp. 547-553. https://doi.org/ 10.1007/978-3-319-25159-2_49

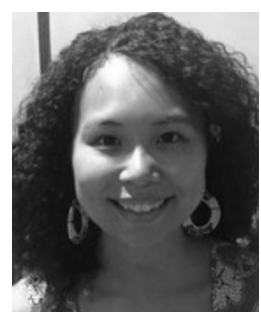

Minami Tsuchiya was born in Fukuoka, then bred in Akita, in Japan. She received her B.E. degree in bio-systems engineering from Yamagata University in 2016. She is currently a student for Ph.D. degree in bio engineering at Graduate School of Science and Engineering, Yamagata University. She also belongs to Innovative Flex Course for Frontier 
Organic Material Systems (iFront), 5 years integrated doctoral course of Yamagata University. Her research interests include vital sensing and analysis, ubiquitous health care system, preventive medicine, self-medication, and information and communications technologies (ICT).

E-mail: tks94610@st.yamagata-u.ac.jp

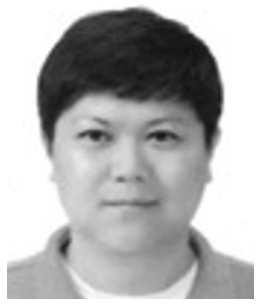

Seung-Il Cho was born in Gwang-ju, Korea, on August 9, 1980. He received his B.S. and M.S. degrees in marine electronics and communication engineering from Mokpo National Maritime University, Korea in 2006 and 2008, respectively. He was an associate research engineer in the $R \& D$ division of Tomato LSI from 2008 to 2010. He received his Ph.D. degree in bio-systems engineering, Graduate School of Science and Engineering, from Yamagata University, Japan in 2013. He is currently an Assistant Professor in the Innovation Center for Organic Electronics, Yamagata University. His research interests include adiabatic dynamic CMOS logic (ADCL), digital/analog integrated circuit design, organic thin film transistor (OTFT) circuit design, organic light emitting diode (OLED) flexible displays, ubiquitous healthcare system, and information and communications technologies (ICT).

E-mail: cho_si@yz.yamagata-u.ac.jp

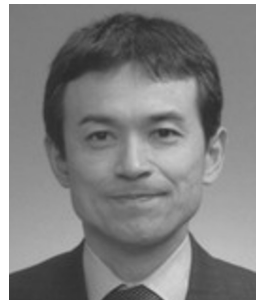

Atsushi Tanaka was born in Niigata, Japan, on September 1st, 1963. He received his B.S. degree in Mathematics from Tohoku University in 1986, and his M.E. and $\mathrm{Ph} . \mathrm{D}$. degree in electrical and communication engineering from Tohoku University in 1988 and 1991, respectively. From 1991, he joined Yamagata University, Yonezawa Japan, and was engaged in reseach on complex systems, especially complex networks recently. He is a member of The Physical Society of Japan, The Information Processing Society of Japan and The Institute of Electronics, Information and Communication Engineers.

E-mail: tanaka@yamagata-u.ac.jp

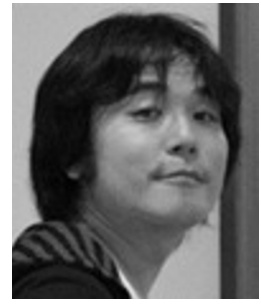

Muneki Yasuda received the doctoral degree (information science) from Tohoku University in 2007. He worked at Tohoku University from 2007 to 2013 as an Assistant Professor. He has worked in Yamagata University as an Associate
Professor since 2013. His research interests are statistical machine learning, data mining, artificial intelligence (AI), signal processing, and statistical mechanics.

E-mail: muneki@yz.yamagata-u.ac.jp

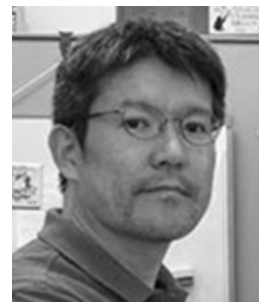

Tomochika Harada received his B.E. degree in Information Engineering from Tohoku University in 1995, and M.E. and $\mathrm{Ph}$.D. degrees in Information Science from Graduate School of Information Sciences, Tohoku University in 1997 and 2000, respectively. From 2000 to 2003, he joined Chuo University as a technical staff. Since 2003, he has joined Yamagata University as an Assistant Professor. His main research interests ultra-low power mixed signal circuits, semiconductor smart sensors based on MOSFET, and the ICT/IoT systems for visualization of various lives. He is a member of IEEJ, IEICE, IEEE and JSAP. He is a recipient of the 2000 IEICE Best Paper Award.

E-mail: tharada@yz.yamagata-u.ac.jp

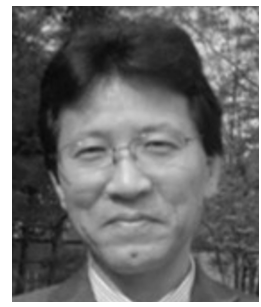

Michio Yokoyama was born in Yamagata, Japan, on March 1st, 1967. He received his B.E. degree in electrical engineering from Yamagata University in 1989, and his M.E. degree in electrical and communication engineering and Ph.D. degree in electronic engineering from Tohoku University in 1991 and 1994, respectively. From 1994 to 2001, he joined the Research Institute of Electrical Communication (RIEC), Tohoku University, Sendai, Japan, where he was engaged in research on the design and development of RF-CMOS devices, such as power amplifier modules for digital cellular phone systems. Since 2001, he has joined Yamagata University, Yonezawa, Japan, and was engaged in research on ubiquitous healthcare system, information and communications technologies (ICT), internet of things (IoT). He is a member of The Japan Institute of Electronics Packaging, The Japan Society of Applied Physics, The Institute of Electrical Engineers of Japan and The Institute of Electronics, Information and Communication Engineers.

E-mail: yoko@yz.yamagata-u.ac.jp 